\title{
The epigenetic component of early-life adversity
}

\section{Benoit Labonté}

\section{Introduction}

The debate on nature versus nurture has already generated lots of discussions. While it has long ago been noted that adverse events occurring early in life increase the risk of developing psychiatric disorders, identifying and characterizing the mechanisms by which these effects are mediated has been challenging. Thus, only recently has evidence allowed researchers to identify potential mechanisms by which the environment may induce behavioral effects. Indeed, epigenetics is now perceived as a potential mechanism by which cells, and by extension complete organisms, may adapt to their environment.

\section{Epigenetics}

Epigenetics refers to the study of the epigenome, the chemical structure surrounding DNA and interfering with normal gene expression without altering the DNA sequence itself. Classically, two main mechanisms have been described: DNA methylation ${ }^{1}$ and/or hydroxymethylation, ${ }^{2}$ and histone modifications. ${ }^{3}$ By interfering with the recruitment of the transcriptional machinery to the DNA, hypermethylation in promoters has classically been associated with transcriptional repression ${ }^{1}$ although more recently, intragenic methylation and hydroxymethylation has been suggested to promote the expression of alternative transcripts and splicing variants. ${ }^{4,5}$ Alternatively, chromatin modifications are known to change chromatin structure (euchromatin/ heterochromatin) and promote or repress access of the transcriptional machinery to the DNA. ${ }^{3}$ Importantly, these transcriptional regulatory mechanisms have been shown to be susceptible to environmental challenges such as early-life adversity, having the consequences of interfering with normal gene expression and to be potentially associated with behavioral modifications during adulthood. ${ }^{6}$

\section{The Impact of Early-Life Stress on Gene Expression}

By definition, early-life adversity is expected to generate a climate of intense stress that may often be persistent in time. As such, one of the systems that is the most likely requested by early-life adversity is the hypothalamicpituitary-adrenergic (HPA) axis, which regulates biological and physiological responses to stress. Early groundbreaking work from the lab of Dr. Michael Meaney showed that variation in maternal care induces changes in the expression of the glucocorticoid receptor (GR17) by altering DNA methylation patterns in the GR regulatory region. Following this elegant piece of work, translational studies showed that similar alterations may also take place in the brain of suicide completers with a history of child abuse. Indeed, McGowan and colleagues showed that abused suicide completers had lower GR 1F (GR 17 homologue) expression levels that were associated with higher DNA methylation levels within GR 1F promoter. Together with other studies on the epigenetic regulation of $G R$ by stress, ${ }^{7,8}$ these findings suggest that the whole GR locus may be poised to epigenetic regulation by environmental stressors such as early-life adversity.

Besides GR, several other genes have been shown to be susceptible to the effects of environment. For instance, other constituents of the HPA axis such as the arginine vasopressine $(A v p)^{9}$ and the corticotrophin releasing hormone $(\mathrm{CRH})^{10}$ genes have been shown to be epigenetically disrupted by stress in rodents. Interestingly, these findings correlate with previous human work showing alterations in the expression of $\mathrm{CRH}$ and pro-opiomelanocortic (pomc) genes in the brain. Similar animal/human correlations can also be made with findings concerning stress-induced regulation of neurotrophins (brain-derived neurotrophic factors; BDNF, glial-derived neurotrophic factor; GDNF) ${ }^{11}$ and their receptors (tyrosine kinase receptor type $\mathrm{B}$; TrkB). Indeed, social stress in mice and rats was shown to interfere with the expression of BDNF in the hippocampus by altering 
histone modification and DNA methylation signatures. ${ }^{12-14}$ In humans, both BDNF and trkB (T1 subtype) were shown to be epigenetically dysregulated in the brain of depressed suicide completers. ${ }^{15-17}$ Several other examples of stressinduced epigenetic alterations in animals and humans have been reviewed elsewhere. ${ }^{6,18}$

\section{Genome wide approaches}

These findings clearly support the involvement of epigenetic mechanisms in mediating the impact of the environment on gene expression and behavior. However, it is unlikely that only a subgroup of genes may be targeted by earlylife adversity as these effects may rather be found across the genome. We recently addressed this question by doing a genome wide screening of promoter DNA methylation patterns in the hippocampus of severely sexually and/or physically abused and non abused suicide completers. ${ }^{19,20}$ Our data showed the presence of hundreds of sites differentially methylated in the promoters of abused and non abused suicides. Importantly, DNA methylation was shown to be inversely correlated with gene expression across the genome.

Note that a proportion of differentially methylated genes was common to the abused and non-abused suicide groups. Although a large proportion were unique to the abused suicides, suggesting that child abuse may impose a certain remodeling of DNA methylation signature in gene promoters. This was confirmed by comparing functions being enriched with differentially methylated genes. Indeed, while cell plasticity was among the most significantly affected functions in the brain of abused suicide completers, functions related to learning and memory processes were unique to the suicide group. Both of these functions are relevant to abuse and suicide, as studies in rodents showed that early life stress decreases adult hippocampal neurogenesis ${ }^{21,22}$ and human studies suggest that learning and memory deficits are symptoms frequently associated with suicidal behaviors. ${ }^{23,24}$ It is also interesting to mention that similar findings have also been reported in peripheral samples of post-traumatic stress disorder (PTSD) ${ }^{25}$ patients and in the prefrontal cortex (PFC) of psychotic and bipolar subjects. ${ }^{26}$

\section{Limitations and future perspectives}

Up to date, most studies focused on brain tissue as this strategy holds the promise to helping improve our understanding of the impact early-life adversity has on brain functions. However, it is still unclear whether these findings will be useful for identifying individuals with specific predispositions toward psychiatric disorders. As such, it will be important to run similar studies in easily accessible tissue such as blood or saliva and to define whether the alterations found in the brain can also be found in peripheral tissue and vice versa. Besides the beneficial effects these studies will have on defining the pathological processes triggered by early-life adversity, it will also allow the identification of predisposed individuals to psychiatric disorders and help in defining better treatment strategies.

Working with animal models helps to generate new working hypotheses. It is, nevertheless, important to determine whether findings can be translated in humans. Presently, working with human samples is challenging given the high level of variability that exists between subjects. No one can assume that each individual went through the same life events, had the same life experience or have the same comorbid disorders. In addition, human postmortem brain studies often have to deal with relatively small sample size given that the resources are rare and precious. It is therefore important to appreciate these limitations, many of which can hardly be addressed with means other than statistics, when considering this type of studies.

Finally, it is important to understand that epigenetics has an integrated and dynamic process involving several components. As such, it will be important to interpret findings in a broader context, by integrating findings from several epigenetic markers (i.e. DNA methylation, hydroxymethylation, several histone modifications) and visualizing their evolution over time. With technology evolving, it will be important to perform more large screening studies looking at the relationship between several markers in the same cell population. In addition, it is important to note that findings summarized previously are actually snapshots of epigenetic signatures at the moment of death. One may expect these alterations to be dynamic over the lifespan. Future studies in animals or humans (peripheral samples) may aim to assess the evolution of these markers in relation to the development of psychiatric conditions.

\section{Conclusion}

There is a growing body of evidence suggesting that the behavioral effects of early-life adversity may be mediated via epigenetic mechanisms. As such, epigenetics may represent an interface on which the environment acts to induce behavioral effect by altering normal gene 
expression. While previous studies convincingly supported this hypothesis, it will be important to launch more largescale comprehensive studies and take advantage of valid and well defined animal models to test and develop new hypotheses. With that being said, it will be necessary to expand this field of research as it has the potential to significantly improve our understanding of mental health and help to define better intervention strategies.

\section{References}

1. Klose RJ, Bird AP. Genomic DNA methylation: the mark and its mediators. Trends Biochem Sci. 2006 Feb;31(2):89-97.

2. Kriaucionis $\mathrm{S}$, Heintz $\mathrm{N}$. The nuclear DNA base 5-hydroxymethylcytosine is present in Purkinje neurons and the brain. Science. 2009 May 15;324(5929):929-30.

3. Kouzarides T. Chromatin modifications and their function. Cell. 2007 Feb 23;128(4):693-705.

4. Maunakea AK, Nagarajan RP, Bilenky M, Ballinger TJ, D'Souza C, Fouse SD, et al. Conserved role of intragenic DNA methylation in regulating alternative promoters. Nature. 2010 Jul 8;466(7303):253-7.

5. Mellen M, Ayata P, Dewell S, Kriaucionis S, Heintz N. MeCP2 Binds to $5 \mathrm{hmC}$ Enriched within Active Genes and Accessible Chromatin in the Nervous System. Cell. 2012 Dec 21;151(7):1417-30.

6. Turecki G, ErnstC, Jollant F, Labonte B, Mechawar N. The neurodevelopmental origins of suicidal behavior. Trends Neurosci. 2012 Jan;35(1):14-23.

7. Labonte B, Yerko V, Gross J, Mechawar N, Meaney MJ, Szyf M, et al. Differential glucocorticoid receptor exon $1(B), 1(C)$, and $1(\mathrm{H})$ expression and methylation in suicide completers with a history of childhood abuse. Biol Psychiatry. 2012 Jul 1;72(1):41-8.

8. McGowan PO, Sasaki A, D’Alessio AC, Dymov S, Labonte B, Szyf M, et al. Epigenetic regulation of the glucocorticoid receptor in human brain associates with childhood abuse. Nat Neurosci. 2009 Mar;12(3):342-8.

9. Murgatroyd C, Patchev AV, Wu Y, Micale V, Bockmuhl Y, Fischer D, et al. Dynamic DNA methylation programs persistent adverse effects of early-life stress. Nat Neurosci. 2009 Nov 8.

10. Elliott E, Ezra-Nevo G, Regev L, Neufeld-Cohen A, Chen A. Resilience to social stress coincides with functional DNA methylation of the $\mathrm{Crf}$ gene in adult mice. Nat Neurosci. 2010 Nov;13(11):1351-3.

11. Uchida S, Hara K, Kobayashi A, Otsuki K, Yamagata H, Hobara T, et al. Epigenetic status of Gdnf in the ventral striatum determines susceptibility and adaptation to daily stressful events. Neuron. $2011 \operatorname{Jan} 27 ; 69(2): 359-72$.

12. Roth TL, Lubin FD, Funk AJ, Sweatt JD. Lasting epigenetic influence of earlylife adversity on the BDNF gene. Biol Psychiatry. 2009 May 1;65(9):760-9.
13. Roth TL, Zoladz PR, Sweatt JD, Diamond DM. Epigenetic modification of hippocampal Bdnf DNA in adult rats in an animal model of post-traumatic stress disorder. J Psychiatr Res. 2011 Feb 8.

14. Tsankova NM, Berton O, Renthal W, Kumar A, Neve RL, Nestler EJ. Sustained hippocampal chromatin regulation in a mouse model of depression and antidepressant action. Nat Neurosci. 2006 Apr;9(4):519-25.

15. Ernst C, Chen ES, Turecki G. Histone methylation and decreased expression of TrkB.T1 in orbital frontal cortex of suicide completers. Mol Psychiatry. 2009 Sep;14(9):830-2.

16. Ernst C, Deleva V, Deng X, Sequeira A, Pomarenski A, Klempan T, et al. Alternative splicing, methylation state, and expression profile of tropomyosin-related kinase B in the frontal cortex of suicide completers. Arch Gen Psychiatry. 2009 Jan;66(1):22-32.

17. Keller S, Sarchiapone M, Zarrilli F, Videtic A, Ferraro A, Carli V, et al. Increased BDNF promoter methylation in the Wernicke area of suicide subjects. Arch Gen Psychiatry. 2010 Mar;67(3):258-67.

18. Labonte B, Turecki $G$. The epigenetics of suicide: explaining the biological effects of early life environmental adversity. Arch Suicide Res. 2010 Oct;14(4):291-310.

19. Labonte B, Suderman M, Maussion G, Lopez JP, Navaro L, Yerko V, et al. Genome-Wide Methylation Changes in the Suicide Brain. American Journal of Psychiatry. 2012;Accepted.

20. Labonte B, Suderman M, Maussion G, Navaro L, Yerko V, Mahar I, et al. Genome-wide epigenetic regulation by early-life trauma. Arch Gen Psychiatry. $2012 \mathrm{Jul} ; 69(7): 722-31$.

21. Malberg JE, Duman RS. Cell proliferation in adult hippocampus is decreased by inescapable stress: reversal by fluoxetine treatment. Neuropsychopharmacology. 2003 Sep;28(9):1562-71.

22. Malberg JE, Eisch AJ, Nestler EJ, Duman RS. Chronic antidepressant treatment increases neurogenesis in adult rat hippocampus. J Neurosci. 2000 Dec 15;20(24):9104-10.

23. Jollant $F$, Bellivier $F$, Leboyer $M$, Astruc $B$, Torres $S$, Verdier $R$, et al. Impaired decision making in suicide attempters. Am J Psychiatry. 2005 Feb;162(2):304-10.

24. Jollant F, Lawrence NL, Olie E, Guillaume S, Courtet P. The suicidal mind and brain: a review of neuropsychological and neuroimaging studies. World J Biol Psychiatry. 2011 Aug;12(5):319-39.

25. Uddin M, Aiello AE, Wildman DE, Koenen KC, Pawelec G, de Los Santos R, et al. Epigenetic and immune function profiles associated with posttraumatic stress disorder. Proc Natl Acad Sci U S A. 2010 May 18;107(20):9470-5.

26. Mill J, Tang T, Kaminsky Z, Khare T, Yazdanpanah S, Bouchard L, et al. Epigenomic profiling reveals DNA-methylation changes associated with major psychosis. Am J Hum Genet. 2008 Mar;82(3):696-711.

\section{Benoit Labonté}

My research interests are to define the molecular impact of stress in the brain and to determine how these effects can affect behaviors and mental health. To do so, I study epigenetic mechanisms, mainly DNA methylation, chromatin modifications, and small RNAs. With my studies, I try to define how genes are influencing behaviors and how changing the function of a gene, by affecting epigenetic mechanisms can have distinct and profound impact of cell functions what can then be translated to a behavioral effect.

My future objectives are to develop my expertise in this highly interesting and stimulating field of research and then get my own lab in order to expand our understanding of stress and mental health. 


\section{Comments on Dialogue Piece \#1 (from HSI Members)}

\section{Comment 1}

This dialogue piece has shed light upon the intricate interplay between genes and the environment, and it has also illustrated one of the mechanisms by which childhood trauma may leave an indelible impression on the human brain.

Providing the vital link between nurture and nature, this exciting piece of research describes how high-throughput techniques can be used to identify epigenetic changes that may arise from abuse events in early childhood.

But this study also raises many interesting questions.

For instance, let us momentarily assume an evolutionary psychology perspective. Through this lens, we can assume that neurological events, like all biological events, are the result of selective pressure. Thus, an epigenetic response to an abuse event might be either (a) an evolutionarily-selected response to abuse or (b) the byproduct of some other evolutionarilyselected response to abuse. It would be very interesting to hear the author's perspective on whether the observed epigenetic responses to abuse confer some form of adaptive benefit - i.e., could the new pattern of gene expression help form a "mental barrier" between the individual and the traumatic events they endured?

At the cellular level, it would be interesting to examine the functional consequences of these epigenetic changes. Are parameters such as neural connectivity, neural distribution, synaptic function, and neurite outgrowth affected by these epigenetic changes? In the absence of patient neuronal samples for such experiments, it is possible that induced pluripotent stem cell-derived neurons, such as those used to model schizophrenia in the laboratory of Dr. Fred Gage at the Salk Institute for Biological Studies, 1 could be used to answer these questions.

From a macroscopic perspective, it would also be very interesting to determine whether epigenetic changes occur at the community level - perhaps in response to a collective trauma such as war or natural disaster. Such studies might also elucidate whether there is an age constraint to this effect, after which epigenetic changes in response to stress or trauma may not be as pronounced.

\section{Brie McKenzie (HSI Managing Editor, Spotlight on Careers)}

\section{References}

1. Brennand KJ, Simone A, Jou J, Gelboin-Burkhart C, Tran N, Sangar S, et al. Modeling schizophrenia using hiPSC neurons. Nature. 2011;473 (7346):221-225.

\section{Comment 2}

"A mind that is stretched by a new experience can never go back to its old dimensions." ${ }^{1}$ While the late American Supreme Court Justice, Oliver Wendell Holmes Jr., may have been speaking metaphorically, the exciting new field of epigenetics sheds light on how experiences and memories, or nurture, can trigger the modification of the functional expression of genes. These impressions can have long-lasting effects on the brain and behaviour of the individual, altering responses to similar events later in life, as well as potentially impacting future offspring. As such, I agree with the author who champions epigenetic research as a way to "define pathological processes triggered by earlylife adversity... identify predisposed individuals to psychiatric disorders and help in defining better treatment strategies" (p. 32, this issue). However, the experience of early-life adversity does not necessarily lead to future psychopathology. Thus I propose expanding the knowledge generation potential of epigenetic research to include an understanding of resiliency.

During the critical period of fetal development there is lots of opportunity for epigenetic variation in response to maternal adversity. But how can health professionals council mothers and families to buffer the long-term effects of adverse pregnancy experiences? In 2004, Canadian researchers discovered the nurturing behaviour of rats resulted in the change of an epigenetic mark at a gene that triggered calm responses to startling in offspring. ${ }^{2}$ Importantly, this epigenetic modification lasted with the animal throughout its life. While humans and rats are markedly different, this finding could mean that attentive interaction between parent and child could dramatically increase resiliency in children that would carry into adulthood. Certainly this area warrants further investigation. Maternal trauma may be uncontrollable, but providing parents with tangible strategies to give children the best possible chance despite experiencing earlylife adversity puts a degree of control back into their hands.

\section{Jennifer Kramer (HSI Reviewer)}

\section{References}

1. Holmes, O.W. The autocrat of the breakfast-table. Boston: M.A. Danahue \& 0 ; 1895 .

2. Weaver, I.C.G., Cervoni, N., Champagne, F.A., D’Alessio, A.C., Sharma, S., Seckl, J.R., et al. Epigenetic programming by maternal behavior. Nature Neuro. 2004;7(8), 847-54. 


\section{Author Response to Comments on Dialogue Piece \#1}

The environment has been known to affect behavior, but it has been challenging to illustrate how this actually happens. Following a growing body of evidence, it is now suspected that epigenetics may be one mechanism in which the organism adapts to environmental pressures. This concept, which can be referred to as resilience, allows the organism to maintain a certain level of physical and psychological functional activity. Related to mental health, resilience may be defined by the capacity of an individual to avoid negative social, psychological and biological consequences of extreme stress that would otherwise compromise their psychological or physical well-being. ${ }^{1}$ Early-life adversity, and particularly childhood abuse, is a major risk factor for the development of mental disorders ${ }^{2}$ but it is important to mention that not all abuse victims develop psychiatric disorders and suicidal behaviors. This suggests that the mechanisms that protect the organism from the threat of abuse may be inefficient in a subgroup of individuals which may then develop mental disorders and potentially suicidal behaviors in the most extreme cases.

Interestingly, certain animal models of stress-induced depression also show a certain animals are non-susceptible to stress. For instance, in one study about $65 \%$ of social defeated mice will show depressive-like behaviors while 35\% won't behave differently from controls; the phenomenon has been interpreted as resilience. ${ }^{3}$ Moreover, these resilient animals don't show the epigenetic alterations found in the susceptible group following stress, but, rather, look like the controls. ${ }^{1}$ These findings suggest that there are epigenetic mechanisms which may be involved in establishing a barrier to the detrimental molecular impact of stress in the brain, and, consequently, protecting the animal from depressive-like behaviors.

Importantly, the detrimental effects of early-life stress seem to be potentially reversed if specific measures are taken into a critical time window. For instance in rats, Michael Meaney and colleagues showed that the pups raised by mothers providing poor maternal care (i.e., low licking and grooming; low LG) behave similarly to pups raised by normal mothers if these pups are cross-fostered to mothers exhibiting high maternal care (high LG) during the first week of life. ${ }^{4}$ Importantly, these measures also reverse the hypermethylation in GR17 promoter found in the low LG group, suggesting that these effects can be reversed if drastic measures are taken before a critical time frame.
Studying resilience in human has been more challenging. For instance, a growing body of evidence suggest that certain personality traits and involvement in religion and community are associated with resilience. ${ }^{5}$ Interesting finding from the group of Dr. Monique Seguin showed that, similar to the findings in rats, life trajectory can be modified if specific measures are taken in early-life (unpublished data). For instance, children with a history of abuse or maltreatment are known to develop life trajectories with poor outcomes, and are more likely to experience mental health problems. However, changing the dysfunctional family environment has been shown in some cases to improve life trajectories. The mediators and moderators involved in these effects are still unknown, but evidence for epigenetics is starting to accumulate. For instance, abuse victims show DNA methylation changes that are not found in the non-abused group. While these changes are associated with changes in gene expression, one may hypothesise that they represent an attempt to modify gene expression patterns in order to adapt to the environmental treats. However, the consequences of these changes appear to be detrimental.

One may hypothesize that these changes may initially have been advantageous for dealing with the occurrence of severe stressors like repeated abuse. However, they may have lost their positive impact as the abuse victims became adults. Indeed, these changes appear to be stable overtime and what was initially advantageous at the moment of the abuse may become disadvantageous in adults. This may be especially true given that these adverse events happen during childhood and adolescence in a period of high plasticity for the developing brain. The long lasting genome-wide changes in DNA methylation may interfere with the establishment of appropriate behavioral and emotional schemes. Consequently, abuse victims are more likely to exhibit stress responses associated with maladaptive emotional and behavioral responses to daily life stressors, which is believed to increase the risk for depression and suicide. ${ }^{6}$

Up to date, it has been challenging to test these hypotheses in the lab and obtain molecular answers using classic methods. However, the development of new approaches may help in obtaining more precise answers. One of these methods involves the use of induced pluripotent stem cells to model depression in a Petri dish. This method consists in taking pluripotent stem cells from a patient with a specific psychiatric illnesses, and treating their cells 


\section{Author Response to Comments on Dialogue Piece \#1}

(4)

0

with various transcription factors in order to make them differentiate into specific cell types, including neuronal progenitors. These techniques have proven to be informative on the cellular alterations that may be taking place in certain psychiatric conditions, including scizophrenia. ${ }^{7}$ It will be interesting to see whether these methods can also be used to study depression and possibly even the molecular and cellular impact of early life stress. However, before getting these answers, it will be important to determine whether inducing by artificial means neuronal differentiation also impacts the epigenome (DNA methylation and chromatin marks), which is known to be highly involved in defining cellular fate across development. But, if this appears to be a minor issue, iPSCs may represent a very powerful model to study the cellular and molecular impact of chronic stress on neurons.

Studying the molecular impact of stress in the brain has highlighted a whole new field of research that may help to clarify certain aspect of stress and psychiatry. Consequently, researchers are starting to study the molecular and epigenetic components potentially involved in post-traumatic stress disorders (PTSD) at a larger scale. For instance, researchers are now studying the impact of civil war in larger community and in war veterans. Others are investigating the long term effects of chronic food deprivation following episodes of famine in certain regions of the world. It will be interesting to follow-up on this work in the coming years, and to continue using animal models, to have deeper insights into the molecular mechanisms regulating the impact of stress on behavior and mental health.

\section{References}

1. Russo, S.J., et al., Neurobiology of resilience. Nat Neurosci, 2012. 15(11): p. $1475-84$.

2. Fergusson, D.M., J.M. Boden, and L.J. Horwood, Exposure to childhood sexual and physical abuse and adjustment in early adulthood. Child Abuse Negl, 2008. 32(6): p. 607-19.

3. Berton, O., et al., Essential role of BDNF in the mesolimbic dopamine pathway in social defeat stress. Science, 2006. 311(5762): p. 864-8.

4. Weaver, I.C., et al., Epigenetic programming by maternal behavior. Nat Neurosci, 2004. 7(8): p. 847-54.

5. Goldstein, A.L., B. Faulkner, and C. Wekerle, The relationship among internal resilience, smoking, alcohol use, and depression symptoms in emerging adults transitioning out of child welfare. Child Abuse Negl, 2013. 37(1): p. 22-32

6. Turecki, G., et al., The neurodevelopmental origins of suicidal behavior. Trends Neurosci, 2012. 35(1): p. 14-23.

7. Brennand, K.J., et al., Modelling schizophrenia using human induced pluripotent stem cells. Nature, 2011. 473(7346): p. 221-5. 